\title{
Klimawandel als Herausforderung für Medizin und Gesundheitswesen
}

\section{René Jaccard}

Dr. med., Mitglied wissenschaftliche Kommission, Klima-Grosseltern gpclimat.ch

\author{
Über den Klimawandel wird dieser Tage viel diskutiert, doch dessen Einfluss auf
} die Gesundheit nur selten angesprochen. Der Autor dieses Artikels rückt das Thema in den Vordergrund und fordert unter anderem, das Gesundheitswesen in der Schweiz $\mathrm{CO}_{2}$-neutral zu gestalten.

Der menschengemachte Klimawandel ist Fakt und mit zahlreichen Beiträgen zu unterschiedlichen Themenbereichen seit Jahren in den Medien präsent. Der Zusammenhang zwischen Klima und Gesundheit jedoch findet nur ganz selten Erwähnung. Nicht besser steht es bei der wissenschaftlichen Literatur: Im Jahr 2017 sind gesamthaft 43000 Publikationen zum Thema Klimawandel erschienen; nur gerade $4 \%$ wiesen einen Zusammenhang zum Thema Gesundheit auf und marginale $<1 \%(n=256)$ befassten sich spezifisch damit [1].

\section{Auswirkungen des Klimawandels auf die Gesundheit}

Die Verbrennung fossiler Brennstoffe setzt das Treibhausgas $\mathrm{CO}_{2}$ frei und ist damit Hauptmotor von Erwärmung und weiteren Klima-Phänomenen. Gleichzeitig entstehen dabei Luftschadstoffe, über deren gesundheitliche Folgen hier bereits kürzlich eingehend berichtet worden ist [2]. Für die Gesundheit relevant sind: extreme Hitzetage, Dürre, Brandkatastrophen, Hurrikane/Taifune, Überschwemmungen, Murgänge, Felsstürze. Extreme Hitze reduziert in einem ersten Schritt die allgemeine Leistungsfähigkeit (siehe auch kürzlich veröffentlichtes BAG-Merkblatt [3]). Ohne prompte Gegenmassnahmen dekompensiert die Temperaturregulation und es kommt zu Hyperthermie, ZNS-Dysfunktion und Multiorganversagen (Überhitzung Hitzschläge - Hitzetote [4]). Die Schäden durch die übrigen erwähnten, aussergewöhnlichen Wetterereignisse können ein solches Ausmass annehmen, dass auch Gesundheitssysteme in reichen Ländern überfordert sind. Als Beispiel dient der Bericht über die verheerenden Waldbrände im November 2018 in Kalifornien, die u.a. zu einem Spitalbrand und chaotischen Zuständen in Verbrennungsabteilungen führten [5]. Als Folge des Klimawandels sinken überdies (trotz Technologieeinsatz) bereits jetzt die Produktivität der Landwirtschaft und wegen Versauerung der Meere die Fischbestände, wodurch Quantität und Qualität der Nahrungsmittelversorgung gefährdet sind. Ebenfalls klimabedingt wandeln sich Spektrum und Auswirkungen Vektor-übertragener Infektionskrankheiten wie z.B. Dengue-Fieber, ZIKA- 
Virusinfektion, Malaria (und weitere); infolge Unterbrechungen der Trinkwasserversorgung häufen sich zudem Cholera-Epidemien [6].

\section{Risikogruppen}

Vulnerabel sind nebst durch chronische Krankheiten geschwächte besonders ältere (>65-jährige) Menschen, was z.B. in der Schweiz die Klima-Seniorinnen zu ihrer aktuell beim Bundesgericht hängigen Klage gegen den Bundesrat veranlasst hat [7]. Ganz speziell gefährdet sind aber auch jüngere und jüngste Menschen: Ein WHO-Bericht legt nahe, dass $>88 \%$ der globalen $\mathrm{Ge}-$ sundheitsschäden $<5$-jährige Kinder betreffen. In Oregon reichten deshalb Jugendliche Klage gegen die USBehörden wegen Untätigkeit ein [8]. Sozioökonomische Faktoren wie Armut, Zugang zu Ressourcen und natürlich, in welcher Weltregion jemand lebt, spielen eine entscheidende Rolle bei der Ausprägung klimabedingter Gesundheitsschäden [1, 6].

\section{Was tun? - 1: Adaptation}

Zum Schutz der Bevölkerung werden weltweit Anpassungen an die veränderten und sich weiter wandelnden Bedingungen bereits heute durchgeführt oder sind in Planung. So müssen z.B. Überwachungssysteme und Empfehlungen betreffend Dengue-Fieber und Malaria abgeändert und neue Erreger (ZIKA-Virus) identifiziert werden; Ressourcen zur Bewältigung klimabedingter Katastrophen werden ausgebaut; Schutzmassnahmen für vulnerable Bevölkerungsgruppen bis hin zu städtebaulichen Massnahmen sind umzusetzen. Dies alles erfolgt national in sehr unterschiedlicher Weise und führt global schätzungsweise zu jährlichen Gesamtkosten von 32,65 Mia. GBP [1].

\section{Was tun? - 2: Klimaschutz}

Je rascher und vollständiger die Dekarbonisierung gelingt, desto grösser wird der Gewinn für Gesundheit und Gesundheitssysteme. Damit werden die künftige, weitere Klimaerwärmung und ihre medizinischen Folgeerscheinungen gebremst. Vor allem aber wird die Luftverschmutzung rasch und massiv reduziert, wodurch sehr bald jährlich 6,5-10 Millionen vorzeitige Todesfälle vermieden werden können [6]. Von den allseits bekannten, weiteren Massnahmen gegen die Klimaerwärmung sind einige mit zusätzlichen Vorteilen für die Gesundheit verbunden: Weitgehend vegetarische Ernährung ist gesund und kann die Treibhausgasemissionen um 20-30\% senken [5]. Fremdenergie-unabhängige Mobilität, d.h. zu Fuss gehen und Velo fahren, fördert ebenfalls die Gesundheit bei gleichzeitiger Senkung der $\mathrm{CO}_{2}$-Emissionen [1].

\section{Der Medizinalsektor als $\mathrm{CO}_{2}$-Emittent und Investor in fossile Energien - Wir könn(t)en entscheidend beitragen}

Wir wissen zwar, dass der globale $\mathrm{CO}_{2}$-Ausstoss durch den Medizinalsektor erheblich ist, unsere Kenntnisse darüber sind aber sehr lückenhaft. Für die USA ergeben die aktuellen Berechnungen knapp 10\% des nationalen Ausstosses, für Australien 7\% [1]. Die australische Studie von 2018 macht Angaben zu CO 2 -Emissionen in einzelnen Sektoren des Gesundheitswesens und weist so auf Möglichkeiten der Entwicklung eines $\mathrm{CO}_{2}$-neutralen Gesundheitswesens hin [9]. In Grossbritannien, als einzigem Land mit systematisch, repetitiv erhobenen Messdaten und einem Programm für Massnahmen zur Reduktion der $\mathrm{CO}_{2}$-Emissionen, konnte zwischen 2007 und 2015 eine Reduktion des $\mathrm{CO}_{2}$-Ausstosses um $11 \%$ auf aktuell $4 \%$ des nationalen Wertes erzielt werden (bei gleichzeitig um 18\% gesteigertem Arbeitsanfall) [1]. Die WHO entwickelt gegenwärtig das Programm «Healthy Planet - Healthy Hospitals - Healthy People» [10]. Aus diesem und den weiteren bereits genannten Quellen lassen sich diverse Empfehlungen zur direkten Reduktion der $\mathrm{CO}_{2}$-Emissionen im Gesundheitswesen ableiten: von der nachhaltigen Gestaltung der Gebäude (v.a. Spitäler) über Abfallvermeidung (Medikamente, Nahrungsmittel, Wegwerfartikel/Plastik) zu Verbesserungen im Transportwesen (grüne Ambulanzen, Velo zur Arbeit) bis hin zur Stärkung der öffentlichen Gesundheit, z.B. durch Propagierung von pflanzlicher Nahrung und Bewegung.

Eine hocheffiziente, indirekte Massnahme zur Senkung von $\mathrm{CO}_{2}$-Emissionen ist die Desinvestition aus fossiler Energie. Von weltweit 428 Milliarden USD, die im Jahr 2017 aus den Fossilen abgezogen wurden, stammten nur gerade 3,28 Milliarden aus Institutionen des Gesundheitswesens. Über die Höhe dieses Betrags in der Schweiz ist nichts bekannt; gemäss Untersuchungen des Bundesamts für Umwelt ergäbe allerdings die aktuelle Art der Investitionen aller Schweizer Pensionskassen eine globale Erwärmung um $4-6^{\circ} \mathrm{C}$ [11].

\section{Was wollen wir uns leisten? - Nichtstun lohnt sich nicht}

Die Transition $\mathrm{zu}$ einer «Null- $\mathrm{CO}_{2}$-Ökonomie» mag zwar konzeptionell äusserst schwierig erscheinen, wird aber schon bald grossen Nutzen bringen, und zwar ganz besonders im Gesundheitsbereich. Wie ausgeführt, ist mit einer raschen Abnahme vorzeitiger 
Todesfälle und assoziierter Morbidität infolge Luftverschmutzung zu rechnen. Zudem wird ein weiterer Anstieg der gegenwärtig infolge Klimaerwärmung jährlich anfallenden Arbeitsausfälle von global 153 Milliarden Stunden mit all ihren ökonomischen Folgen gebremst [1]. Und zwischen 2030 und 2050 können gemäss WHO weltweit jährlich 250000 Hitzetodesfälle bei älteren Menschen vermieden werden [6], um nur die wichtigsten Beispiele zu nennen. Immer wieder werden die Kosten als Grund gegen den Übergang auf eine Netto-Null- $\mathrm{CO}_{2}$-Energiegewinnung und effiziente Energiesparmassnahmen ins Feld geführt.

Die gegenwärtigen, globalen ökonomischen KostenNutzen-Rechnungen gehen jedoch von einer neutralen, möglicherweise sogar positiven Gesamtbilanz aus, wenn der Faktor klimaabhängige Gesundheit voll einbezogen wird [6]. Wenn wir also jetzt, im Jahr 2019, das richtige Szenario starten mit dem Ziel, die Klimaerwärmung bis 2050 zwischen $+1,5$ und $2,0^{\circ} \mathrm{C}$ zu halten, könnten wir im Endeffekt Kosten sparen.

Die Zeit hierfür wird allerdings knapp! Als Ärztinnen und Ärzte sind wir ungebunden und können uns in Klimafragen voll für das Wohl unserer Patientinnen und Patienten sowie unseres Gesundheitswesens einsetzen: Wir brauchen mit den nötigen Kompetenzen ausgestattete Beauftragte für Nachhaltigkeit in allen Gesundheitsdepartementen, medizinischen Fakul-

\section{Das Wichtigste in Kürze} setzt werden. Dafür werden mit den nötigen Kompetenzen

Dr. med. René Jaccard rene.rjaccard[at]

datacomm.ch täten, Krankenkassen, Spitälern, Praxisgemeinschaften. National müssen unsere $\mathrm{CO}_{2}$-Emissionen systematisch evaluiert und ein Reduktionsplan mit dem Ziel $\mathrm{CO}_{2}$-Neutralität des Gesundheitswesens bis 2030 allerspätestens 2050 in Gang gesetzt werden. Die im Gesundheitswesen geäufneten Guthaben, z.B. von Pensionskassen, sollen ab sofort betreffend ihrer Auswirkungen auf $\mathrm{CO}_{2}$-Ausstoss und Klima klassiert und im Sinne der Gesundheitsförderung platziert werden.

Bildnachweis

(c) Dmitriy Melnikov | Dreamstime.com

\section{Literatur}

1 Watts N, Amann M, Arnell N, et al. The 2018 report oft the Lancet Countdown on health and climate change: shaping the health of nations for centuries to come. Lancet. 2018;392:2479-514.

2 Peters A, Hoffmann B, Brunekreef B, et al. Die Rolle der Luftschadstoffe für die Gesundheit. Schweiz Ärzteztg. 2019;100(23-24):796-9.

3 BAG-Bulletin 24/2019.

4 Epstein Y, Yanovich R. Heatstroke. N Engl J Med. 2019;380:2449-59.

5 Solomon CG, LaRocque RC. Climate Change - A Health Emergency. N Engl J Med. 2019;380:209-11.

6 Haines A, Ebi K. The Imperative for Climate Action to Protect Health. N Engl J Med. 2019;380:263-73.

7 https://klimaseniorinnen.ch/warum-wir-klagen/

8 Salas RN, Jacobs W, Perera F. The case of Juliana v. US - Children and the Health Burdens of Climate Change. N Engl J Med. 2019;380:2085-7. 9 www.thelancet.com/planetary-health; Vol 2, January 2018.

10 www.who.int/globalchange/publications/healthcare settings/en/

11 https://www.bafu.admin.ch/bafu/de/home/themen/klima/ fachinformationen/klima-und-finanzmarkt.html
- Die Auswirkungen des Klimawandels auf die Gesundheit sind vielfältig, dieTransition zu einer «Null- $\mathrm{CO}_{2}$-Ökonomie» würde speziell im Gesundheitsbereich grossen Nutzen bringen.

- Der globale $\mathrm{CO}_{2}$-Ausstoss durch den Medizinalsektor ist erheblich, das derzeit durch die WHO entwickelte Programm "Healthy Planet - Healthy Hospitals - Healthy People» enthält diverse Empfehlungen zur direkten Reduktion der $\mathrm{CO}_{2-}$ Emissionen im Gesundheitswesen.

- National müssen $\mathrm{CO}_{2}$-Emissionen systematisch evaluier und ein Reduktionsplan mit dem Ziel $\mathrm{CO}_{2}$-Neutralität des Gesundheitswesens bis 2030, allerspätestens 2050, in Gang geausgestattete Beauftragte für Nachhaltigkeit in allen Gesundheitsdepartementen, medizinischen Fakultäten, Krankenkassen, Spitälern, Praxisgemeinschaften gebraucht.

\section{L'essentiel en bref}

- Les effets du changement climatique sur la santé sont multiples et le passage à une économie "zéro $\mathrm{CO} 2$ " serait particulièrement bénéfique dans le domaine de la santé.

- Les émissions mondiales de $\mathrm{CO} 2$ du secteur médical sont considérables. Le programme "Healthy Planet - Healthy Hospitals - Healthy Peoplen actuellement mis au point par I'OMS contient plusieurs recommandations pour les réduire.

- Au niveau national, les émissions de $\mathrm{CO} 2$ doivent être systématiquement évaluées et un plan de réduction visant la neutralité carbone du système de santé en 2030 ou au plus tard en 2050 doit être mis en œuvre. II faudra donc, dans I'ensemble des départements de santé, facultés de médecine, caisses d'assurance-maladie, hôpitaux et cabinets collectifs, des chargés du développement durable disposant des compétences nécessaires.

- Ein ökonomisch verträglicher Uebergang zur fossilfreien Energiegewinnung ist möglich, eine entsprechende Investitionspolitik des Medizinalsektors kann dazu beitragen.
- Une transition économiquement supportable vers la production d'énergie entièrement non fossile est possible et une politique d'investissement correspondante dans le secteur médical peut y contribuer. 